\title{
FWI estocástico: Inversão da forma de onda com os métodos GA e PSO
}

\author{
Daniel E. Revelo ${ }^{1,2}$, Rodrigo de S. Santos ${ }^{1}$ e Oscar M. Ladino ${ }^{1}$ \\ ${ }^{1} \mathrm{HPC} / \mathrm{SENAI} / \mathrm{CIMATEC}, \quad{ }^{2} \mathrm{CPGG} / \mathrm{UFBA}$ \\ Copyright 2018, SBGf - Sociedade Brasileira de Geofísica. \\ Este texto foi preparado para a apresentação no VIII Simpósio Brasileiro de Geofísica \\ Salinópolis, 18 a 20 de setembro de 2018. Seu conteúdo foi revisado pelo Comitê \\ Técnico do VIII SimBGf, mas não necessariamente representa a opinião da SBGf ou de \\ seus associados. É proibida a reprodução total ou parcial deste material para propósitos \\ comerciais sem pr'evia autorização da SBGf.
}

\section{Resumo}

Um dos principais objetivos e desafios da geofísica de exploração de oléo e gás é identificar regiões com grandes reservas de hidrocarboneto. O uso de técnicas do método sísmico tem possibilitado o imageamento destas regiões, facilitando a localização de novos campos e melhoria no entendimento de campos maduros. Diversas técnicas de imageamento estão presentes no método sísmico, sendo que a maioria destas técnicas tem performance intimamente ligada à qualidade de um bom modelo de velocidades da subsuperficie. Por esta razão, estimar um bom modelo de velocidades tem se apresentado como um pré-requisito paro o imageamento sísmico. Atualmente, o método de inversão da forma de onda (FWI - Full Waveform Inversion) tem ganhado destaque devido a sua capacidade de estimar modelos de velocidades com mais alta resolução que os métodos convencionais até então empregados. Na forma mais convencional, a FWI faz uso de métodos determinísticos no processo de minimização da diferença entre os dados observados e calculados. Estes métodos têm como principal desvantagem o fato de serem métodos de inversão de escopo local e, assim, necessitarem de um modelo inicial relativamente acurado para convergir. Uma outra abordagem da FWI pode ser feita utilizando métodos não determinísticos no processo de otimização, a qual será nomeada de FWI não convencional. No presente trabalho, para este fim, implementou-se os métodos: Algoritmo Genético (GA - Genetic algorithm) e Optimização por enxame de Partículas (PSO - Particle Swarm Optimization). Os resultados obtidos permitiram avaliar a influência da quantidade de parâmetros da inversão no resultado final, assim como o desempenho dos algoritmos na FWI unidimensional.

\section{Introdução}

Atualmente na indústria do petróleo o método sísmico é o mais empregado, isso devido a sua capacidade de registrar reflexões associadas a refletores situados a alguns quilômetros de profundidade. Esse método tem como objetivo final fornecer uma "imagem" da subsuperfície, para o qual é necessário o processamento de dados registrados na superfície. Neste contexto, a etapa de análise das velocidades sísmicas tem fundamental importância, uma vez que tal etapa fornece as velocidades usadas para migrar os dados sísmicos registrados em superfície para a sua verdadeira posição em profundidade. Este fato torna a determinação do campo de velocidades uma das principais etapas do processamento sísmico, e, por isso, muitos esforços têm sido feitos no desenvolvimento de novas técnicas para a estimativa do campo de velocidades. Dentre as diversas abordagens na estimativa do campo de velocidade, a técnica CDP (Common Depth Point) foi durante muitos anos a base para a geração do campo de velocidades (Yilmaz, 2001). Está técnica, entretanto, baseia-se em aproximações (p. ex., modelo de camadas plano paralelas) e, portanto, em situações com geologia complexas, que não atendem as aproximações exigidas pelo processamento CDP, não permite obter imagens com a exatidão requerida, dificultando o processo de interpretação. Desta forma, novas técnicas surgiram para atender está demanda, com destaque para o método de inversão da forma de onda (Tarantola, 1984), possibilitando a obtenção de imagens mais fiéis da subsuperfície, além da utilização do próprio modelo de velocidades como uma ferramenta na interpretação geológica. O objetivo da FWI é a obtenção de um modelo de velocidades, cuja modelagem sobre este forneça um dado sintético mais próximo possível do dado real observado, o que inclui os tempos de trânsito, a fase e a amplitude no sinal sísmico (dos Santos e Pestana, 2015). Este processo é feito partindo de um modelo inicial, seguido de sucessivas atualizações desse até que um critério de parada seja alcançado.

A FWI é um problema de otimização do tipo não linear, multidimensional e caracterizado por funções objetivas complexas com muitos mínimos locais. Existem essencialmente duas categorias de métodos de inversão que podem ser usados na FWI: aquelas baseadas em métodos determinísticos, nos quais é empregado o computo do gradiente da função custo (p. ex., o método Gauss-Newton, gradiente conjugado). Estes métodos são atraentes pois convergem rapidamente para um mínimo, no entanto, eles precisam de um modelo inicial de boa qualidade para encontrar o mínimo global. Além disso, eles exigem o cálculo das derivadas de primeira e/ou segunda ordem da função objetivo, um processo que pode ser computacionalmente exigente no caso de modelos com uma grande quantidade de parâmetros. Por outro lado, tem-se a categoria dos algoritmos baseados em métodos estocásticos tais como: GA, PSO e Very Fast Simulated Annealing, (Sen e Stoffa, 1995); que realizam uma busca no espaço global de modelos e não requerem nenhum cálculo das derivadas da função objetivo. Diferentemente dos determinísticos, esses métodos conseguem estimar o modelo de mínimo global mesmo sem a existência de um bom modelo inicial. Essas características tornam sua aplicação particularmente adequada quando não se tem uma estimativa inicial que guarde características do modelo de minimo global. A desvantagem dos métodos estocásticos é que eles geralmente precisam de muitas 
avaliações do modelo para convergir, o que dificulta sua aplicação em inversões com muitos parâmetros a serem determinados e/ou exigindo modelagens diretas que são caras em termos computacionais. Aproveitando as vantagens dessas duas abordagens, diversos trabalhos têm proposto abordagens híbridas no tratamento de problemas inversos com muitos parâmetros de inversão (de S. Santos e Porsani, 2017; van Leeuwen et al., 2011; Sajeva et al., 2016). No presente trabalho é apresentada uma versão não convencional da FWI unidimensional, com o objetivo de obter um modelo de velocidades ótimo, e com essa finalidade dois tipos de métodos estocásticos são implementados: GA e PSO, tornando o processo da FWI numa inversão de escopo global. Diversos testes serão apresentados a fim de avaliar o desempenho da FWI com os algoritmos GA e PSO quando o número de parâmetros é aumentado ou o espaço de busca é incrementado.

\section{Modelagem Direta, Problema Inverso e FWI}

A maioria dos problemas da geofísica podem ser alocados como problema direto ou inverso. Em ambas as situações existe um relacionamento entre as propriedades ou parâmetros físicos de um modelo, m (p. ex., densidade, resistividade ou velocidade), e os dados de um levantamento ou modelagem geofísica, d. De maneira geral, os processos de modelagem e inversão destinguem-se quanto ao papel das variáveis $\mathbf{d}$ e $\mathbf{m}$. No problema direto, $\mathbf{m}$ representa a variável independente e d a dependente, por outro lado, no problema inverso os papéis são trocados. Isto é, quando os parâmetros são conhecidos e deseja-se obter $\mathbf{d}$, estamos resolvendo o problema direto, ou simplesmente realizando uma modelagem geofísica. Quando d é conhecido e deseja-se estimar $\mathbf{m}$, nesse caso trata-se de resolver um problema inverso (Fig. 1). Em ambas as situações, sempre deve existir um relacionamento teórico que conecte $\mathbf{d}$ com $\mathbf{m}, 0$ qual pode ser descrito conforme a relação abaixo:

$$
\mathbf{d}=\mathbf{G}(\mathbf{m}),
$$

onde, G é o operador que guarda a relação teórica entre os parâmetros do modelo e os dados. No caso da FWI, d representa os traços do sismograma (dado sísmico), G é o operador de modelagem com base na equação da onda e m o perfil ou campo de velocidades. Algumas vezes é possível encontrar um operador de inversão $\mathbf{G}^{-1}$ que resolve o problema inverso, porém na $\mathrm{FWI}$ isso não é possível. Assim, o problema inverso deve ser tratado como um problema de otimização de uma função objetivo $Q(\mathbf{m})$. Desta forma, a solução é encontrada em um número finito de iterações, onde, a cada iteração, um modelo $\mathbf{m}_{k+1}$ é encontrado por meio de uma pertubação $\Delta \mathbf{m}_{k}$ no modelo corrente $\mathbf{m}_{k}$, conforme a Eq. (2). Então, realiza-se a modelagem em $\mathbf{m}_{k+1}$ e o dado calculado é comparado com o dado observado.

$$
\mathbf{m}_{k+1}=\mathbf{m}_{k}+\Delta \mathbf{m}_{k}
$$

A Eq. (2) apresenta a forma padrão de atualização do modelo corrente em um problema de inversão. Nos problemas clássicos da $\mathrm{FWI}$, o termo $\Delta \mathbf{m}_{k}$ é função do gradiente da função objetivo, porém aqui usaremos os métodos GA e PSO para este fim, os quais não requerem nenhum cálculo das derivadas da função custo. De acordo com o exposto anteriormente, pode-se perceber que em um problema de otimização dois processos de extrema importância são necessários: a atualização do modelo corrente, que neste trabalho será realizada com os métodos estocásticos, e a modelagem do dado calculado, o qual será feita com base na solução da equação da onda acústica unidimensional.

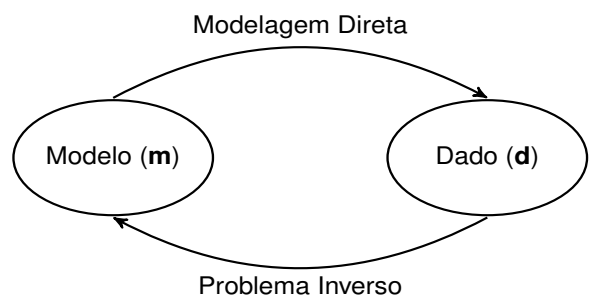

Figura 1: Relação entre $\mathbf{d}$ e $\mathbf{m}$ nos problemas direto e inverso.

\section{Função objetivo e equação da onda}

O processo de inversão baseado em otimização tem como princípio minimizar (ou maximizar) uma função objetivo. Existem várias definições desta função objetivo, porém no presente trabalho será empregada aquela que busca minimizar a soma dos quadrados dos resíduos entre os dados observados $\mathbf{d}_{o b s}$ e calculados $\mathbf{d}_{c a l}$, definida por:

$$
Q(\mathbf{m})=\left\|\mathbf{d}_{o b s}-\mathbf{d}_{c a l}\right\|_{2},
$$

onde, $\mathbf{d}_{c a l}$ é modelado pela equação da onda em função do modelo de velocidades corrente, ou seja, $\mathbf{d}_{c a l}=\mathbf{G}\left(\mathbf{m}_{k}\right)$, onde $\mathbf{m}_{k}=c$, em que $c$ representa o perfil de velocidades do modelo. A cada iteração, é necessário calcular $\mathbf{d}_{c a l}$ e para isso deve-se definir o operador de modelagem G responsável para tal. Neste trabalho, a modelagem sísmica será feita através da equação da onda acústica 1D que é definida como:

$$
\frac{\partial^{2} P(z, t)}{\partial z^{2}}=\frac{1}{c^{2}} \frac{\partial^{2} P(z, t)}{\partial t^{2}},
$$

onde, $P(z, t)$ é o campo de pressão, $c \rightarrow c(z)$ o modelo de velocidades, $z$ é a variável espacial e $t$ a variável temporal. Para resolver a Eq. (4) foi implementada a técnica de diferenças finitas na aproximação das derivadas espaciais e temporais (Lines et al., 1999).

\section{Algoritmo Genético}

O Algoritmo Genético é um método usado na busca da solução ótima de problemas de otimização, que tem suas bases ligada aos principios da evolução biológica de um sistema natural (população). Este método baseiase no fato de que seres (modelos) mais adaptados a um dado ecossistema tendem a sobreviver e propagar suas características para gerações futuras. No GA os modelos candidatos a solução de um problema inverso são tratados como membros de uma população, sobre a qual são realizadas operações que simulam processos biológicos. As operações realizadas permitem a obtenção de gerações futuras e, assim, espera-se que em um número finito de gerações a população de modelos convirja para a solução ótima do problema. O GA difere 
dos métodos de inversão determinísticos nos seguintes aspectos:

- Os GA trabalham com um conjunto de parâmetros a ser otimizado de uma forma codificada, podendo ser binária por analogia aos cromossomos (Sen e Stoffa, 1995).

- Os GA usam diretamente a informação a ser otimizada (função objetivo), não utilizam derivadas ou outros conhecimentos auxiliares (dos Santos et al., 1995).

- Os GA utilizam regras de transição probabilística e não regras determinísticas, e isso evita a exigência de convergência local.

O uso do GA envolve algumas etapas que são chamadas de operadores genéticos, sendo as principais:

1. Geração: A populçao de modelos é gerada por meio de pertubações num modelo inicial $\mathbf{m}_{0}$.

2. Codificação: Codifica-se os parâmetros do modelo.

3. Seleção: Seleciona-se os modelos que possuem maior aptidão com o problema.

4. Cruzamento: Combina-se os modelos selecionados na etapa anterior gerando novos.

5. Mutação: Etapa que gera de forma aleatória modificações em membros da população. Isto mantém diversidade na populaçãio e evita convergência prematura.

O algoritmo é executado por várias iterações até alcançar um critéio de parada ou retornar ao passo 3.

\section{Otimização por enxame de partículas}

Proposto por Kennedy e Eberhart (1995), o PSO é uma técnica de otimização global inspirada no comportamento social de organismos biológicos, mais especificamente na habilidade de algumas espécies de animais de trabalhar em conjunto para localizar boas regiões com fontes de alimento, tais como o comportamento de bandos de pássaros ou peixes na procura de alimentos, pelo qual pode ser incluída no conjunto de algoritmos evolutivos. Neste algoritmo cada modelo representa uma partícula, e o enxame de partículas explora o espaço de possíveis soluções para otimizar uma determinada função de custo, que em nosso caso está relacionada ao problema inverso.

O processo evolutivo do algoritmo baseia-se na atualização das velocidades das partículas do enxame ao longo do tempo gerando a modificação do posicionamento destas. O método PSO utiliza um população de partículas, onde a posição de cada partícula $\left(\left\{x_{i}\right\}_{i=1}^{p}\right)$ consiste de um vetor de $M$ dimensões cujos componentes correspondem aos parâmetros a serem determinados. Cada partícula se movimenta no espaço de modelos com uma velocidade específica $\left\{v_{i}\right\}_{i=1}^{p}$. A magnitude e direção da velocidade das partículas dependem de três fatores: a velocidade atual de cada partícula $\left\{v_{i(k)}\right\}_{i=1}^{p}$ (fator de inércia), a sua melhor posição ao longo de todo o processo até o momento, definida por $\left\{g_{i}\right\}_{i=1}^{p}$ (fator cognitivo) e a posição da melhor partícula de todo o enxame ao longo do processo, definida por $G$ (fator social). No início do PSO, as partículas são inicializadas em posições aleatórias no espaço de busca segundo uma distribuição de probabilidade uniforme. Em seguida, a cada iteração $k$, as velocidades $\left(v_{i}\right)$ e as posições $\left(x_{i}\right)$ das partículas são atualizadas de acordo com as seguintes fórmulas:

$$
\begin{aligned}
& v_{i}(k+1)=\omega \times v_{i}(k)+a_{1} \times a_{l o c} \times \frac{\left(g_{i}-x_{i}(k)\right)}{\Delta t} \\
&+a_{2} \times a_{g l o b} \times \frac{\left(G-x_{i}(k)\right)}{\Delta t} \\
& x_{i}(k+1)=x_{i}(k)+v_{i}(k+1) \Delta t,
\end{aligned}
$$

onde, $\omega$ é a inércia, $a_{l o c}$ e $a_{g l o}$ são as acelerações local e global, respectivamente, $a_{1}$ e $a_{2}$ são números randômicos entre 0 e 1 , e $\Delta t$ é passo temporal de valor unitário.

No PSO original, proposto por Kennedy e Eberhart, foi verificado que a velocidade das partículas alcançavam rapidamente valores muito altos, especialmente para partículas longe de seu líder cognitivo e do seu líder social. Para fugir deste problema, geralmene é adotada a estratégia de limitar cada componente do vetor velocidade a um valor máximo $v_{\max , j}$, definido como uma fração do espaço de busca (Engelbrecht, 2007), da forma

$$
v_{\max , j}=\alpha \times\left(x_{\max , j}-x_{\min , j}\right),
$$

em que $x_{\max , j}$ e $x_{\min , j}$ são respectivamente os valores máximos e mínimos do espaço de busca na dimensão $j$, e $\alpha$ é um parâmetro tal que $\alpha \in(0,1]$. Originalmente, 0 algoritmo PSO foi utilizado com $\left(\omega, a_{l o c}, a_{g l o}\right)=(1,2,2)$ (Kennedy e Eberhart, 1995), mas esta formulação pode fazer com que as partículas oscilem em torno de seu centro, impedindo o sistema de alcançar a convergência total. Para garantir a convergência, Clerc (1999) desenvolveu uma abordagem para equilibrar o compromisso entre exploração e explotação, na qual a equação da velocidade é modificada para:

$$
\begin{aligned}
v_{i}(k+1)=\phi \times\left(v_{i}(k)\right. & +a_{1} \times a_{l o c} \times\left(g_{i}-x_{i}(k)\right) \\
& \left.+a_{2} \times a_{g l o b} \times\left(G-x_{i}(k)\right)\right),
\end{aligned}
$$

onde $\phi$ é um termo de constrição que depende das duas acelerações:

$$
\phi\left(a_{l o c}, a_{g l o b}\right)=\frac{2}{\left|2-a_{l o c}-a_{g l o b}-\sqrt{\left(a_{l o c}+a_{g l o b}\right)^{2}-4\left(a_{l o c}+a_{g l o b}\right)}\right|} .
$$

Pela descrição feita sobre o algoritmo PSO, é aconselhável, além de definir uma velocidade máxima $v_{\max }$ para todas as partículas, selecionar $a_{l o c}+a_{g l o b} \geq 4$ (Clerc e Kennedy, 2002), isto com o intuito de garantir a convergência do método. Em geral, o algoritmo PSO básico, utilizado em problemas sem restrições, pode ser definido conforme os seguintes passos:

1. Inicializar o conjunto de partículas com velocidades igual a zero e posições aleatoriamente distribuídas dentro do espaço de busca;

2. Avaliar a função objetivo de cada partícula da população;

3. Atualizar a melhor posição de cada partícula individualmente e a melhor posição do bando;

4. Atualizar a posição de cada partícula no tempo $k+1$ baseado na posição e velocidade no tempo $k$;

5. Repetir os processos de 2 a 4 até que uma condição de parada seja satisfeita. 


\section{Resultados}

Neste trabalho usou-se a FWI não convencional para obter modelos de velocidades 1D, tendo como entrada o traço sísmico sintético obtido a partir da modelagem sobre o modelo verdadeiro. Durante o processo de modelagem sísmica alguns parâmetros foram fixos, tais como a frequência de pico $f_{\text {peak }}=11 \mathrm{~Hz}$, a taxa de amostragem na profundidade $\Delta z=10 \mathrm{~m}$ e a taxa de amostragem temporal de $\Delta t=1 \mathrm{~ms}$. Os demais parâmetros foram alterados de acorco com a necessidade do experimento. Com base nesta estrátegia realizaram-se diversos experimentos, buscando-se avaliar quais são as influências do número de parâmetros de inversão, e da faixa de busca, na convergência da FWI não convencional com base nos métodos globais GA e PSO.

É importante salientar que os testes foram feitos em modelos sintéticos, o que tornou possível avaliar o desempenho dos métodos com modelos de diferentes configurações. Algumas dessas configurações extrapolam limites existentes na realidade, como o limite da profundidade de investigação que em situações reais dificilmente ultrapassam $10 \mathrm{~km}$, no entanto, neste trabalho realizamos testes em modelos $1 \mathrm{D} \mathrm{com}$ até $33 \mathrm{~km}$ de profundidade, contudo isso não invalida a importância dos resultados.

\section{Variação da quantidade de parâmetros}

Nesta seção apresenta-se os resultados realizados em seis diferentes configurações de modelos de velocidades, sendo que cada modelo possui diferente quantidade de camadas e de amostras na profundidade. Nesta abordagem, o espaço de busca foi mantido fixo numa faixa de $\pm 250 \mathrm{~m} / \mathrm{s}$ em relação ao modelo verdadeiro (Fig. 3), alterando apenas alguns dos parâmetros, como apresentado na Tabela 1. Na Figura 2 é apresentado o dado sintético e calculado para o modelo de 40 camadas, onde observa-se que o dado foi reconstruido corretamente. A Figura 3 mostra os resultados da inversão para os seis modelos com até 150 parâmetros, como pode ser observado, obteve-se uma convergência satisfatória, deixando em evidência a possibilidade do uso de tal abordagem em problemas $2 \mathrm{D}$ para a estimativa de macro modelos que servem como entrada para FWI convencional.

\section{Variação da faixa de busca}

Neste segundo experimento foi mantida uma configuração de modelo fixa, fazendo-se variar a faixa de busca dos parâmetros, e dessa forma avaliou-se a convergência dos métodos GA e PSO. Para esse experimento foi usado o modelo de 40 camadas, com uma população de 400 modelos e fazendo a faixa variar desde $\pm 250 \mathrm{~m} / \mathrm{s}$ até $\pm 1000 \mathrm{~m} / \mathrm{s}$ em relação ao modelo verdadeiro. Os demais parâmetros foram mantidos (Tabela 1), porém realizouse os experimentos fazendo 1000 iterações. Na Figura 4 é apresentado o resultado da inversão para a faixa de $\pm 750 \mathrm{~m} / \mathrm{s}$. A Figuras 5 mostra o comportamento da função objetivo, onde pode-se ver que para uma faixa de até $\pm 1000 \mathrm{~m} / \mathrm{s}$, existe boa similaridade entre os modelos verdadeiros e invertidos. Esse resultado mostra que, para uma situação em que se busca o modelo ótimo num raio de busca de até $\pm 1000 \mathrm{~m} / \mathrm{s}$, é possivel obter convergência com os métodos GA e PSO.

Esse resultado também mostra a tendência global do método, isso porque quando se estipula uma faixa de $\pm 1000 \mathrm{~m} / \mathrm{s}$, estamos cobrindo uma região de até $2000 \mathrm{~m} / \mathrm{s}$. Em situações reais, quando se realiza inversão, quase sempre se conhece alguma informação a priori, como um campo de velociades de NMO, por exemplo, e essa informação é suficiente para se estipular uma faixa de busca menor ou similar a $\pm 1000 \mathrm{~m} / \mathrm{s}$, o que mostra que a metodologia estudada torna possível, de forma satisfatória, a obtenção do modelo de velocidades.

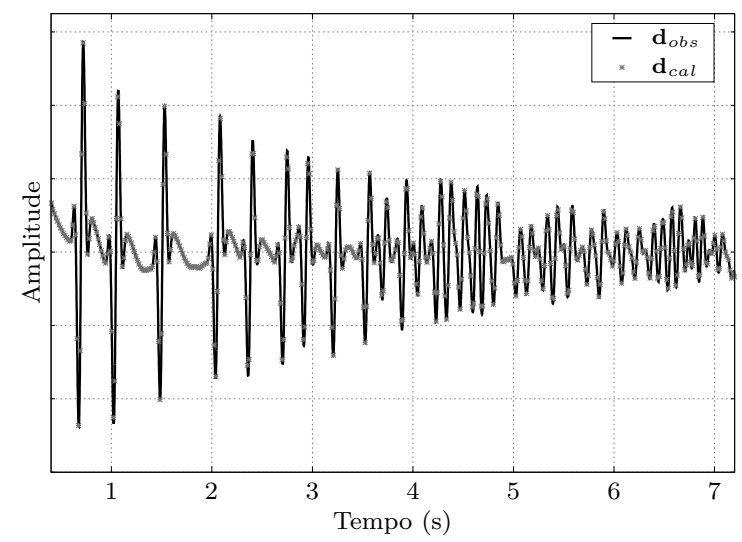

Figura 2: Sismograma observado e calculado para o modelo de velocidade de 40 camadas. A modelagem sísmica foi realizada segundo os parâmetros da Tabela 1. A onda direita foi eliminada para melhorar a visualização dos eventos.

\section{Conclusão}

Neste trabalho realizou-se a FWI 1D não convencional usando os métodos estocásticos GA e PSO. Com o alvo de estudar a convergência dos métodos em função das quantidades de parâmetros e das faixas de busca, foram realizados diferentes experimentos. Os resultados apresentados no primeiro experimento mostram que os métodos convergiram de forma satisfatória para o caso FWI 1D de até 150 parâmetros. Esse resultado permite concluir que a metodologia desenvolvida pode ser estendida para o caso 2D, se for usado a estratégia de redução de parâmetros, como realizado por Sajeva et al. (2016).

$\mathrm{Na}$ segunda classe de testes foi usado um modelo de 40 camadas, e nesse caso a faixa de busca foi sucessivamente aumentado até atingir $\pm 1000 \mathrm{~m} / \mathrm{s}$, onde pode-se observar nas Figuras 4 e Figuras 5 que até para este valor máximo obteve-se convergência, o que evidencializa o sucesso da abordagem proposta. Este segundo resultado enfatiza a natureza global do GA e PSO. A partir desses resultados, conclui-se que o uso de FWI 1D não convencional pode ser feita de forma satisfatória com base nos algoritmos globais estudados, e em trabalhos futuros, pretende-se estender o uso desta abordagem para modelos 2D. 


\begin{tabular}{|c|c|c|c|c|c|c|}
\hline \multicolumn{6}{|c|}{ Parâmetros dos experimentos - modelagem e inversão } \\
\hline \hline Modelo & M10 & M20 & M40 & M70 & M100 & M150 \\
\hline \hline Camadas & 10 & 20 & 40 & 70 & 100 & 150 \\
\hline Amostras $z(n z)$ & 500 & 500 & 1000 & 1600 & 2300 & 3340 \\
\hline Iterações $N_{i t}$ & 100 & 300 & 300 & 400 & 700 & 1000 \\
\hline População $N_{p}$ & 200 & 200 & 300 & 600 & 800 & 1200 \\
\hline Amostras $t(n t)$ & 2800 & 3850 & 7200 & 10570 & 16880 & 22150 \\
\hline
\end{tabular}

Tabela 1: Informações da modelagem e inversão para cada uma das configurações de modelos de velocidades.

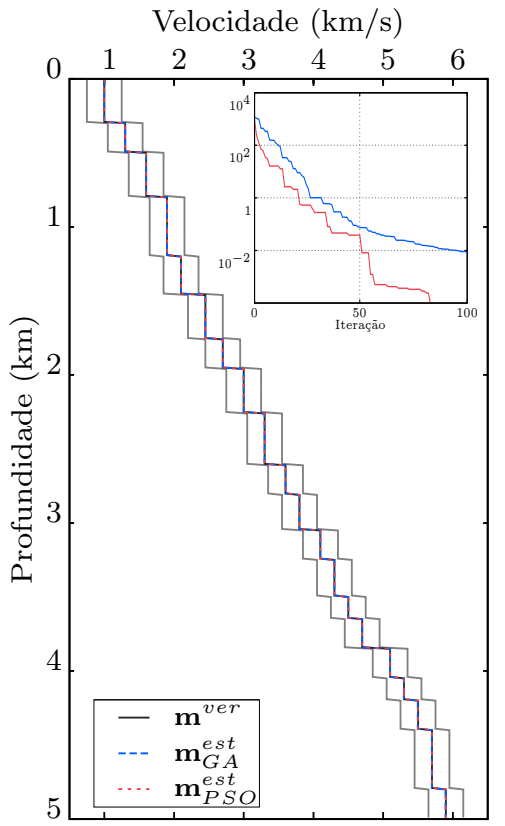

a) 10 camadas

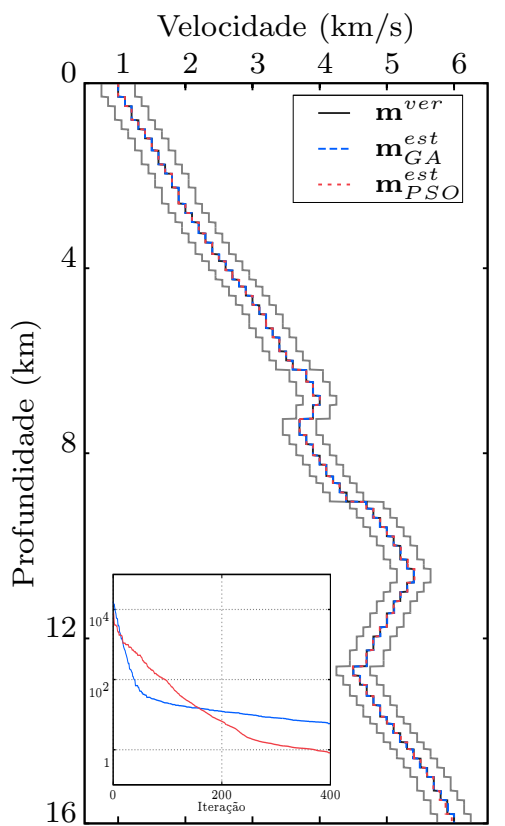

d) 70 camadas

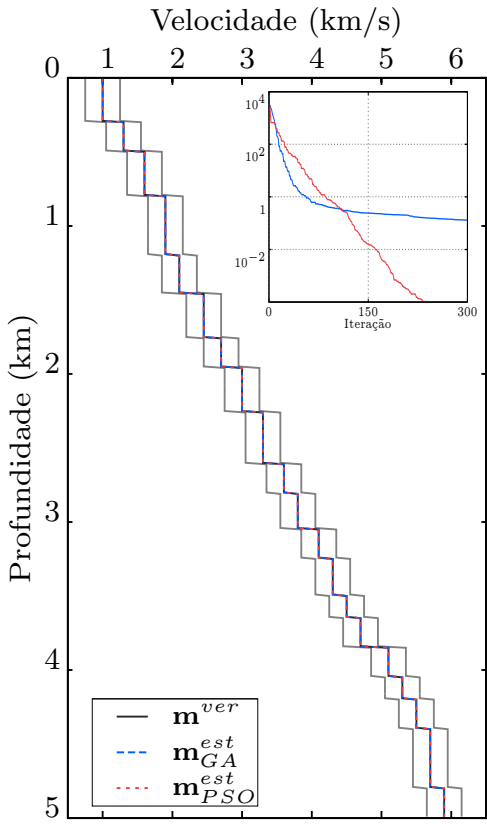

b) 20 camadas

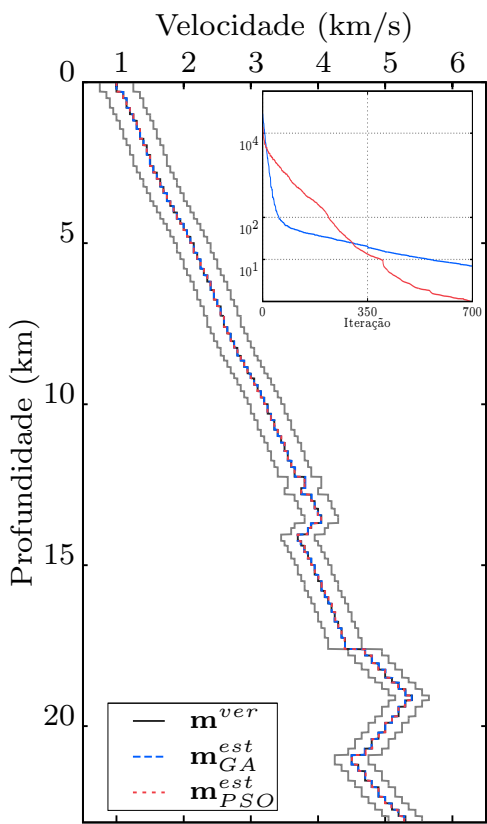

e) 100 camadas

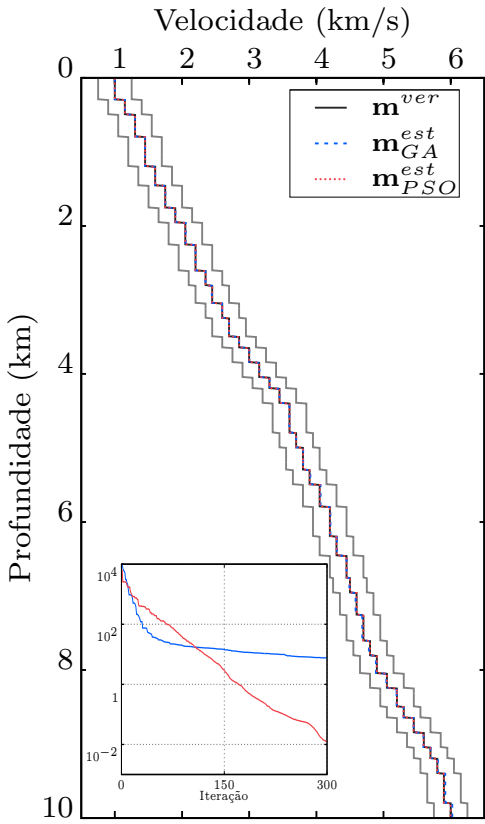

c) 40 camadas

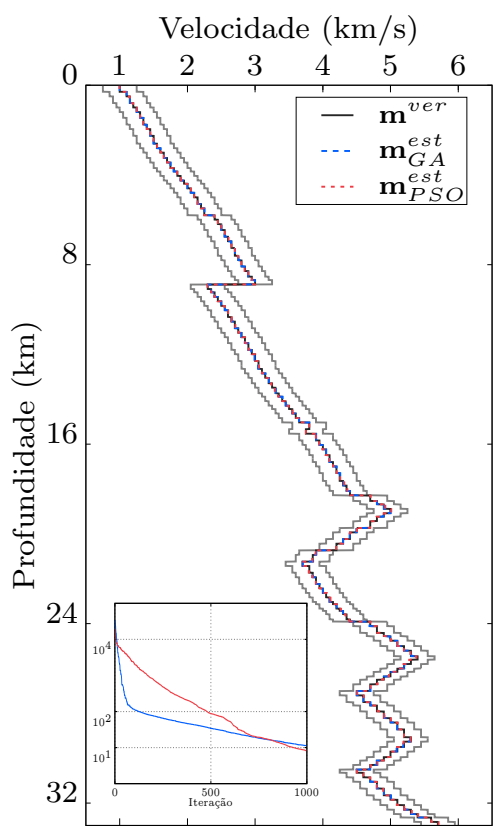

f) 150 camadas

Figura 3: Resultados da FWI 1D não convencional para as diversas configurações do modelo de velocidades. Nos pares de figuras são apresentadas o comportamento da função objetivo e o modelo estimado a cada teste. 


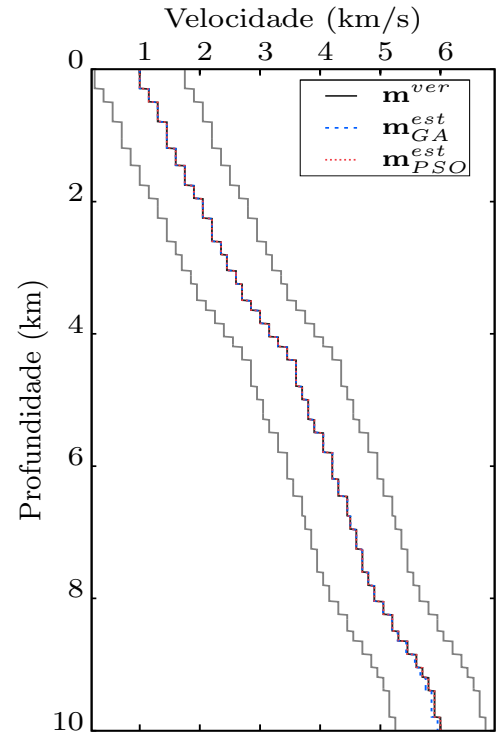

Figura 4: FWI 1D não convencional para o modelo de 40 camadas com faixa de busca de $\pm 750 \mathrm{~m} / \mathrm{s}$. Além dos modelos verdadeiro e estimados pela FWI com os métodos AG e PSO, também os limites da faixa de busca em color cinza ao longo do modelo são mostrados.

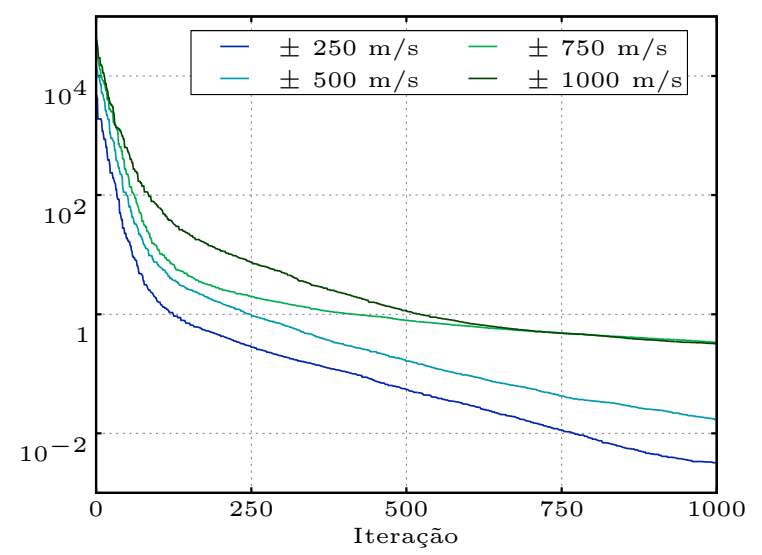

a) Algoritmo genético

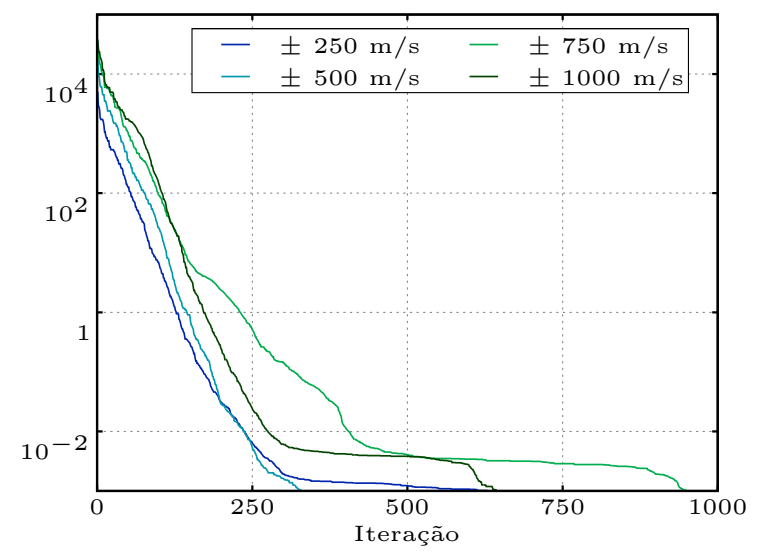

b) Optimização por enxame de partículas

Figura 5: Comportamento da função objetivo dos métodos de optimização implementados para cada uma das faixas propostos.

\section{Acknowledgements}

Os autores agradecem à SHELL e ao Centro de Supercomputação do SENAI CIMATEC para Inovação Industrial pela permissão em publicar a presente pesquisa. Este projeto recebeu financiamento da Agência Nacional do Petróleo (ANP) e da Empresa Brasileira de Pesquisa e Inovação Industrial (EMBRAPII).

\section{Referências}

Clerc, M., 1999, The swarm and queen: towards a deterministic and adaptive particle swarm optimization.: Proceedings of the IEEE Congress on Evolutionary Computation., 1951-1957.

Clerc, M. e J. Kennedy, 2002, The particle swarm-explosion, stability, and convergence in a multidimensional complex space.: IEEE Transactions on Evolutionary Computation., 6, 58-73.

de S. Santos, R. e M. J. Porsani, 2017, Obtaining interval velocities using an adaptive hybrid inversion multiscale approach.: Presented at the Expanded Abstracts, Fifteenth International Congress of the Brazilian Geophysical Society.

dos Santos, A. e R. Pestana, 2015, Time-domain multiscale full-waveform inversion using the rapid expansion method and efficient step-length estimation: Geophysics, 80, R203-R216.

dos Santos, P., M. Porsani, e P. Stoffa, 1995, Inversăo elástica 1D utilizando algoritmos genéticos: Presented at the 4th International Congress of the Brazilian Geophysical Society.

Engelbrecht, A., 2007, Computational inteligence an introduction: John Wiley Sons, The Atrium, Southern Gate, Chichester, West Sussex PO19 8SQ, England, 2007.

Kennedy, J. e R. Eberhart, 1995, Particle swarm optimization.: IEEE International Conference on Neural Networks., 4, 1942-1948.

Lines, L., R. Slawinski, e R. Bording, 1999, A recipe for stability of finite-difference wave-equation computations: Geophysics, 64, 967-969.

Sajeva, A., M. Aleardi, E. Stucchi, N. Bienati, e A. Mazzotti, 2016, Estimation of acoustic macro models using a genetic full-waveform inversion: Applications to the Marmousi model: Geophysics, 81, R173-R184.

Sen, M. e P. Stoffa, 1995, Global optimization methods in geophysical inversion: Cambridge University Press.

Tarantola, A., 1984, Inversion of seismic reflection data in the acoustic approximation: Geophysics, 49, 12591266.

van Leeuwen, T., M. Schmidt, M. Friedlander, e F. Herrmann, 2011, A hybrid stochastic-deterministic optimization method for waveform inversion: Presented at the 73rd EAGE Conference and Exhibition incorporating SPE EUROPEC 2011.

Yilmaz, O., 2001, Seismic data analysis, vol. 10 of investigations in geophysics: SEG, Tulsa. 